\title{
Influencia de la marea y factores antropogénicos sobre la calidad fisicoquímica de aguas costeras en Nuquí, Chocó
}

\section{Influence of tide and factors anthropogenic on the physicochemical quality of coastal waters in Nuquí, Chocó}

\author{
Zoraida Quesada Martínez ${ }^{1}$, Yirlesa Murillo Hinestroza², Lady Vargas Porras ${ }^{3}$
}

\section{Resumen}

Se hizo un análisis de la calidad fisicoquímica del agua en una zona costera de Nuquí, considerando las concentraciones de oxigeno disuelto (OD), $p H$, salinidad, conductividad, turbiedad, nutrientes (fosfatos, nitritos ynitratos), coliformesfecales, sólidos disueltos totales, DBO5, DQO, grasas y aceites. Se realizaron mediciones in situ y muestreos en marea baja y alta, en estaciones ubicadas en la desembocadura de fuentes hídricas que reciben descargas de aguas residuales, en el área de su intercepción con la zona costera y directamente sobre la zona costera. Lo anterior con el fin de determinar los efectos de las perturbaciones antrópicas sobre la calidad fisicoquímica de las aguas costeras y evaluar su estado como ecosistema prioritario para el establecimiento de grupos biológicos. Para ello, se hicieron mediciones in situ y análisis en laboratorio de variables fisicoquimicas y los resultados se compararon con normas y estándares de calidad de agua para la preservación de la fauna y la flora acuática. Se encontró que a pesar de que los parámetros monitoreados no presentaron concentraciones que indiquen una restricción para el desarrollo de los organismos acuáticos, existen áreas puntuales que de acuerdo con las presiones de las actividades urbanas pueden afectar el desarrollo de los mismos por el aporte de altas cargas contaminantes de coliformes fecales, $D Q O, D B O 5$, fosfatos, nitritos nitratos, grasas y aceites que son vertidos en las fuentes hídricas continentales que vierten sus aguas a la zona costera.

Palabras clave: Aguas costeras, Calidad de agua, Nuquí, Perturbaciones antrópicas, Variables fisicoquímicas.

\begin{abstract}
An analysis of the quality of the water was in a coastal area of Nuqui, considering the concentrations of dissolved oxygen (OD), pH, salinity, conductivity, turbidity, nutrients (phosphates, nitrites and nitrates), fecal coliform, total dissolved solids, DBO5, DQO, fats and oils. In situ and sampling at low and high tide, at stations located at the mouth of water sources receiving wastewater discharges in the area of interception with the coastal zone and directly on the coastal area measurements they were made. This in order to determine the effects of human disturbance on the physico-chemical quality of coastal waters and evaluate their status as a priority for the establishment of biological groups ecosystem. To do this, in situ measurements and analysis they were made in laboratory physicochemical variables and the results were compared with standards and water quality standards for the preservation of wildlife and aquatic flora. It was found that although the monitored parame-
\end{abstract}

Ingeniera Ambiental y Sanitaria, Especialista en Gestión Integral de Cuencas Hidrográficas. Investigadora del Instituto de Investigaciones Ambientales del Pacífico (IIAP), Quibdó, Colombia. e-mail: zoraidaquesada35@gmail.com

2 Ingeniera Ambiental. Instituto de Investigaciones Ambientales del Pacífico (IIAP), Quibdó, Colombia. e-mail: yirdavid@hotmail.com Ingeniera Ambiental y Sanitaria. Especialista en Gestión del Recurso Hídrico. Investigadora de Proyectos Especiales, Instituto de Investigaciones Ambientales del Pacífico (IIAP), Quibdó, Colombia. e-mail: lvargas@iiap.org.co

Recibido: 15 de enero de 2014

Aprobado: 4 de marzo de 2014 


\section{Bioetnia Volumen 11, 2014}

ters did not show concentrations indicating a constraint for the development of aquatic organisms, there are specific areas according to the pressures of urban activities can affect their development for the contribution of higher pollutant loads of fecal coliforms, $D B O_{5}, D Q O$, phosphates, nitrates, nitrites, fats and oils that are deposited on inland water sources that drain into the coastal zone.

Keywords: Anthropogenic disturbances, Coastal waters, Nuqui, Physico-chemical variables, Water quality.

\section{Introducción}

Desde el punto de vista del ambiente marino-litoral, se reconocen en la región del Chocó Biogeográfico, dentro de las ocho subregiones ecogeográficas, definidas con base en los rasgos morfológicos de la costa, la amplitud de la plataforma continental, la influencia del drenaje de los ríos en las aguas costeras y la distribución de los principales hábitats litorales, los cuales son de sur a norte: Tumaco, Sanquianga, Naya, Gorgona, Buenaventura, Baudó y Chocó, todas en el Pacífico, y Darién en el Caribe (Banco de Occidente 2014). De otro lado, las huellas de las actividades humanas se encuentran en todos los océanos; aunque las áreas oceánicas aún se encuentran relativamente limpias, en las aguas menos profundas, particularmente bahías y estuarios, se presentan graves conflictos; allí se suma la contaminación de aguas, sedimentos y organismos, los hábitats han sido destruidos $\mathrm{y}$ es donde ha tenido lugar el mayor agotamiento de pesquerías y recursos (Burke et al. 2001).

Como la vida en el ambiente acuático está determinada por la calidad y las características del agua, todo cambio en ella involucra cambios en la biota y en su composición; de allí la importancia de conocer las condiciones del agua en cuanto a sus características fisicoquímicas y biológicas, debido a que se constituye en muchos casos, en un cuerpo receptor de materiales contaminantes como los residuos sólidos y líquidos que se generan en un centro poblado. Más aún, cada vez se ha hecho claro que las actividades realizadas en tierra son las principales fuentes de contaminación en los mares y costas. Sin embargo, también se reconoce que no solo la contaminación es responsable del deterioro de los océanos, también lo son el daño físico directo a los ecosistemas y la sobrexplotación de los recursos (GESAMP 2001).

De acuerdo con Garay (2004), al igual que en el Caribe, las aguas servidas urbanas han sido identificadas como uno de los mayores contaminantes que afectan el medio costero del Pacífico colombiano. Aproximadamente $87.211 \mathrm{~m} /$ día de estas aguas sin ningún tipo de tratamiento son arrojadas a las zonas costeras, representadas en residuos líquidos de cerca 374.631 habitantes de las principales ciudades y asentamientos humanos costeros. El 91,5\% de la carga orgánica y bacteriana lo aportan las ciudades costeras de los departamentos de Valle del Cauca y Nariño, sobre todo las ciudades de Buenaventura y Tumaco, cuya población sumada representa $86,6 \%$ del total de la población costera del Pacífico colombiano.

La zona costera de Nuquí está ubicada en la costa pacífica del Chocó Biogeográfico, constituye gran importancia desde el punto de vista de desarrollo turístico en esta zona, la cual está influida por los vertimientos directos de residuos sólidos y líquidos. Como consecuencia de la erosión causada por el río Ancachí y por el océano Pacífico, el sistema de alcantarillado urbano convencional presenta múltiples puntos de vertimientos, donde cada uno de ellos arroja pequeños caudales producto de grupos reducidos de viviendas, motivo por el cual, no existe un emisario, interceptor o colector de importancia que permita tomar un aforo representativo de un sector apreciable del sistema de alcantarillado. Por otra parte, en marea alta, las aguas marinas ingresan a parte del sistema en particular a los tramos finales produciendo rebosamiento de las aguas residuales (Aguas y Aguas de Pereira 2012).

En este estudio se determinó, mediante monitoreo in situ y toma de muestras para análisis de laboratorio, la calidad fisicoquímica del agua mediante la determinación de las concentraciones de parámetros como oxígeno disuelto (OD), pH, salinidad, conductividad, turbiedad, DBO5, DQO, nutrientes, grasas y aceites, además de la determinación de organismos indicadores de contaminación fecal para determinar la presencia de patógenos en las aguas como coliformes fecales. Lo anterior con el objetivo de analizar el estado del recurso como elemento importante en la conservación y preservación de la biota acuática en los ecosistemas costeros de Nuquí, además del análisis de la influencia de las mareas (baja y alta) y las perturbaciones de origen antropogénico sobre las concentraciones de los parámetros fisicoquímicos mencionados.

\section{Métodos}

Área de estudio. El municipio de Nuquí se encuentra localizado en la parte occidental del departamento del Chocó, sobre la vertiente occidental de la serranía del Baudó en la costa pacífica colombiana a una distancia de $184 \mathrm{~km}$ de la capital del departamento; su cabecera municipal es Nuquí y se encuentra ubicado en el centro del territorio, sobre la costa del golfo de Tribugá en la desembocadura de los ríos Nuquí y Ancachí (Aguas y Aguas de Pereira 2012). La caracterización fisicoquímica y biológica, estuvo determinada por la ubicación de 4 puntos de muestreo sobre el río Ancachí, su intercepción con el río Nuquí y más 200 metros sobre la bahía. A estas dos fuentes se vierten 
Marea y factores antropogénicos. Z Quesada et al.

Tabla 1

Descripción y localización de puntos de monitoreo

Bahía de Nuquí

\begin{tabular}{cll}
\hline Punto & \multicolumn{1}{c}{ Descripción } & Georeferenciación \\
\hline P1 & Río Ancachí & \\
P2 & Intercepción río Nuquí-Ancachí-Bahía & $5^{\circ} 42^{\prime} 22.3^{\prime \prime}-77^{\circ} 16^{\prime} 6.8^{\prime \prime}$ \\
P3 & +200 metros de la estación 2 & $5^{\circ} 47^{\prime} 33.6^{\prime \prime} 32.77^{\circ} 16^{\prime} 29.3^{\prime \prime}$ \\
P4 & +200 metros de la estación 3 & $5^{\circ} 42^{\prime} 54.7^{\prime \prime}-77^{\circ} 16^{\prime} 30.9$ “ \\
\hline
\end{tabular}

todas las aguas residuales provenientes de pozos sépticos y otros sistemas de evacuación de aguas domésticas de la zona urbana del municipio. Además, hay una alta presión por la erosión que se presenta en la ronda hídrica del río Ancachí, lo que posiblemente trae como consecuencia un aporte significativo de sedimentos, por lo cual se requirió la determinación de las concentraciones de sólidos en el cuerpo de agua (IIAP 2014) (Tabla 1, Figuras 1 y 2).

Métodos. Para evaluar la calidad del agua a través del análisis de las variables fisicoquímicas y biológicas se seleccionaron puntos de muestreos cada $200 \mathrm{~m}$ desde la desembocadura de los ríos Nuquí y Ancachí hasta la zona costera; en cada punto se hicieron mediciones in situ y toma de muestras en superficie y a un metro de profundidad.

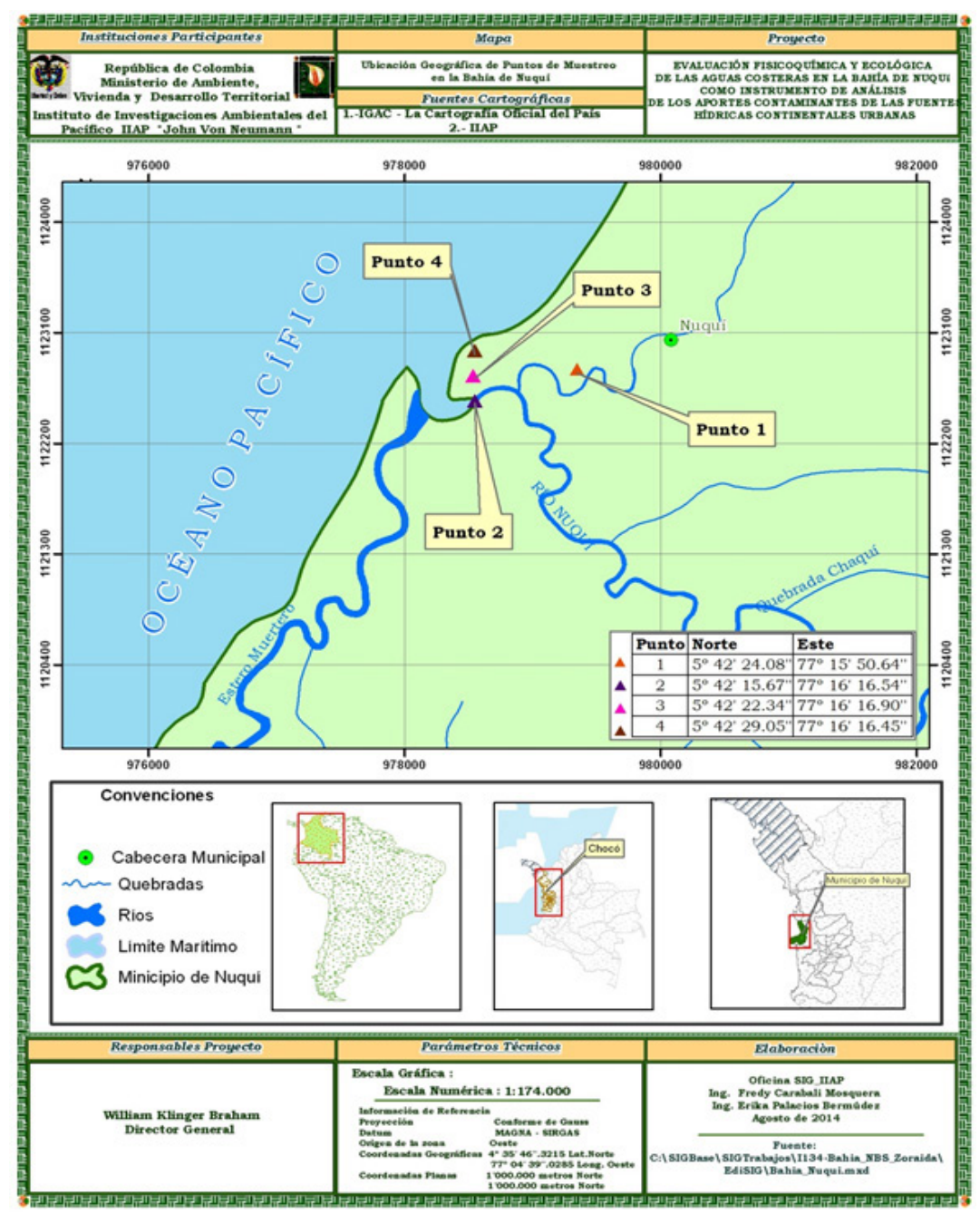

Figura 1. Puntos de muestreo bahía de Nuquí. 


\section{Bioetnia Volumen 11, 2014}

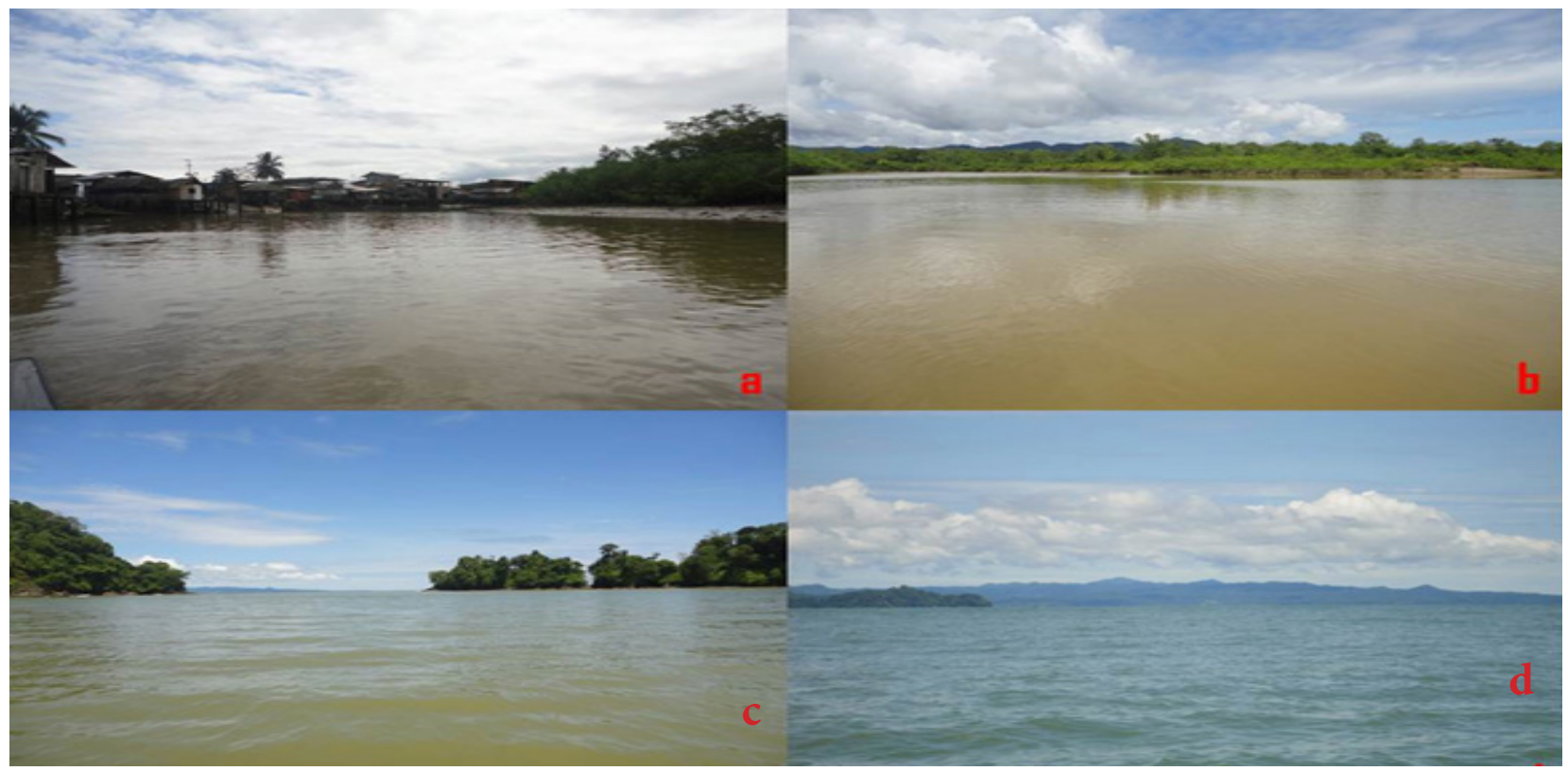

Figura 2. a. Río Ancachí. b. Intercepción río Nuquí-Ancachí-Bahía. c. +200 metros de la estación 2. d. +200 metros de la estación 3.

Estos muestreos se realizaron durante marea baja y alta, para dar respuesta al comportamiento de las variables en la dinámica de flujo. Las variables determinadas in situ fueron: temperatura, oxígeno disuelto(OD), conductividad, turbiedad, fosfatos, nitritos, nitratos, $\mathrm{pH}$ y salinidad, utilizando un colorímetro portátil HACH 850 y un multiparámetro YSI PROFESIONAL PLUS QUICK 1700/1725. La toma de muestra para análisis en laboratorio de parámetros como coliformes fecales, DQO, sólidos disueltos totales,
DBO5, grasas y aceites, se hizo mediante la utilización de frascos previamente esterilizados, cumpliendo con los protocolos para toma y recepción de muestras establecidos por el laboratorio de aguas de la Corporación Autónoma para el Desarrollo Sostenible del Chocó (CODECHOCO). Además, se utilizó un GPS para la georeferenciación de cada punto (Figura 3).

Para análisis de los efectos de las perturbaciones antrópicas sobre la calidad de agua, se hizo una comparación

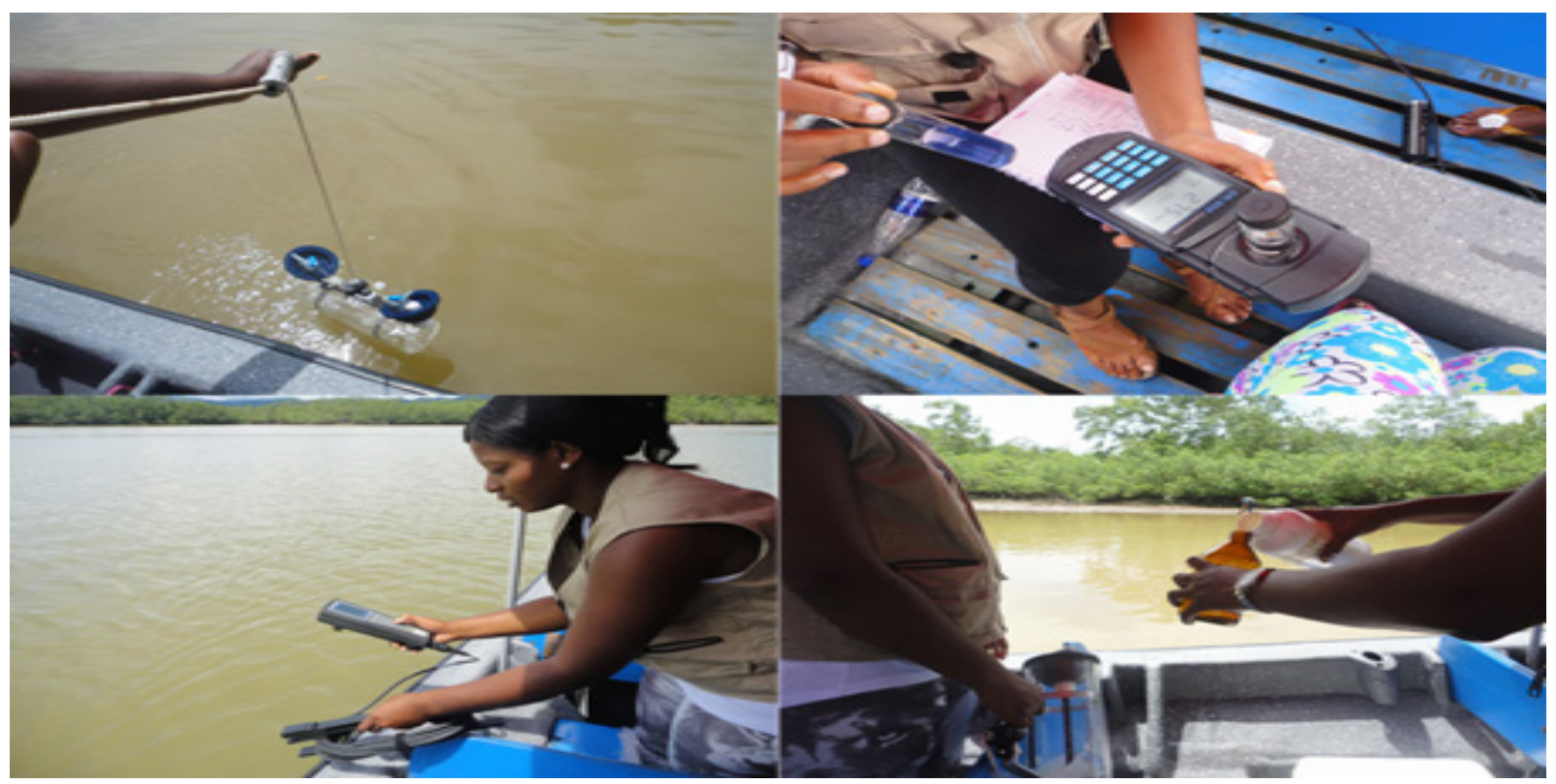

Figura 3. Monitoreo y toma de muestras bahía de Nuquí. 


\section{Marea y factores antropogénicos. Z Quesada et al.}

de los datos obtenidos con los estándares de calidad para la preservación de la fauna y la flora acuática establecidos por la normatividad nacional e internacional, así como con los datos arrojados por otras investigaciones de calidad de agua en fuentes superficiales y las observaciones realizadas en campo sobre el estado del agua, su dinámica de flujo y su interacción con componentes biológicos.

\section{Resultados}

Caracterización fisicoquímica y análisis de la calidad de agua. Los resultados de monitoreo de la calidad fisicoquímica de la bahía de Nuquí se presentan en la Tabla 2. De acuerdo con los resultados obtenidos se pudo establecer que durante el tiempo de monitoreo tanto en marea alta como en marea baja, los valores de temperatura en la zona costera de Nuquí, permanecieron casi constantes, oscilando entre $29,7^{\circ} \mathrm{C}$ en marea baja y $29,2^{\circ} \mathrm{C}$ en marea alta, evidenciándose los mayores registros en los puntos de monitoreo 3 y 4 . Pese a las pocas variaciones, los datos arrojados indican aguas cálidas que favorecen el desarrollo de la vida acuática, porque esta variable juega un papel importante en los procesos fisiológicos, tales como la respiración microbiana responsable en muchos de los procesos de auto-purificación en los cuerpos de agua superficiales (Boyd 1982). Es importante destacar que se evidencia la influencia de las condiciones mareales en los resultados de esta variable física, existiendo una relación directa con la salinidad, la cual se obtuvo en mayor proporción en mareas altas con un valor de $28.1 \mathrm{mg} / 1 \mathrm{de} \mathrm{Cl}$ en el punto 3 , decreciendo rápidamente hacia la profundidad, lo que pudo estar relacionado con una mayor presencia de radiación solar recibida sobre la superficie, la influencia del viento y una mayor estratificación por calentamiento en el área de la desembocadura de la bahía (Obeso et al. 2012).

En relación con el $\mathrm{pH}$, este se mantuvo casi constante oscilando entre 7,6 y 8,2; no se presentaron variaciones significativas en términos de mareas ni en monitoreo a superficie o a profundidad, encontrándose dentro de lo establecido por el Decreto 1594 de 1984, el cual constituye un rango de 6,5-8,5 para la preservación de fauna y flora en aguas costeras y estuarinas. Estos valores indican aguas casi neutras con una tendencia ligeramente alcalina, lo cual sugiere que el ecosistema presenta una estabilización de un potencial de hidrógeno que resulta favorable para el desarrollo de los procesos biológicos vitales y la permanencia de las especies en su interior.

Para el OD los datos oscilaron entre $6,18 \mathrm{mg} / \mathrm{l}$ en marea alta y 6,43 en marea baja, datos que de acuerdo con el Decreto 1594 de 1984 favorecen la preservación de la flora y la fauna, porque para este tipo de ecosistemas este valor debe ser igual o superior a $4 \mathrm{mg} / \mathrm{l}$. Las mayores concentraciones de OD se obtuvieron en los puntos de monitoreo sobre el río Ancachí, evidenciándose que pese a los vertimientos de origen antrópico que recibe, se mantienen condiciones favorables para la biota acuática. Adicional a lo anterior, se conserva un esquema en la disminución de las concentraciones de OD en los monitoreos a profundidad (un metro) reflejándose el impacto que causa la carga orgánica de los residuos líquidos proveniente de la zona urbana del municipio de Nuquí.

La conductividad osciló entre $945,48.330 \mu \mathrm{s} / \mathrm{cm}$ y 372 , $47.403 \mu \mathrm{s} / \mathrm{cm}$, para la marea baja y alta respectivamente, siendo los valores más altos, los reportados en la estaciones $+200 \mathrm{~m}$ sobre la zona costera, lo que obedece a una mayor influencia del agua del mar en la bahía, las cuales contienen grandes cantidades de iones y sales disueltas que incrementan esta variable en este tipo de ecosistemas y que además tienen una estrecha relación con variables como sólidos disueltos y la turbiedad, esta última oscilando entre 51 FAU, 1025 FAU y 1, 82 FAU, para marea baja y alta, siendo los puntos más altos las estaciones 1 y 2 correspondientes al río Ancachí y a la intersección entre este, el río Nuquí y la bahía, lo que puede estar relacionado con vertimientos puntuales de desechos sólidos y líquidos de origen antrópico, además del aporte de sólidos provenientes de los desprendimiento de tierra, productos de la erosión hídrica sobre el cauce del río Ancachí. Al respecto Derisio (1992) expresa que los valores de conductividad y solidos disueltos en el agua, dependen de la presencia o no de sales y residuos sólidos en la fuente hídrica (Figura 4).

Los sólidos disueltos totales (SDT), se obtuvieron en mayor concentración en las estaciones de monitoreo 3 (intercepción ríos Anchachí, Nuquí y bahía +200), tanto a profundidad como a superficie en marea baja; adicionalmente se obtuvo una concentración significativa a profundidad de la estación 4 (200 m de la estación 3). También, en marea alta, se obtuvieron las mayores concentraciones en estas estaciones a profundidad. Es de anotar que todas las aguas naturales contienen sólidos disueltos en mayor o menor proporción; a esta propiedad también se le llama salinidad del agua. Las sales disueltas se presentan como cationes y aniones y al ser partículas cargadas eléctricamente conducen la electricidad, lo que indica una relación directa entre los SDT, la salinidad y la conductividad, que se refleja en los datos obtenidos en las estaciones de monitoreo, donde se observa que a mayor salinidad, mayor conductividad y por lo tanto, mayor concentración de sólidos disueltos totales lo cual se evidencia en los datos obtenidos del monitoreo en marea alta, que además muestra un aumento en las concentraciones de estos parámetros en la estación 2, debido a la influencia del agua de mar en la misma (Figura 5).

Los contenidos de materia orgánica evaluados a partir de la DBO5 comprendida como la cantidad de oxígeno 


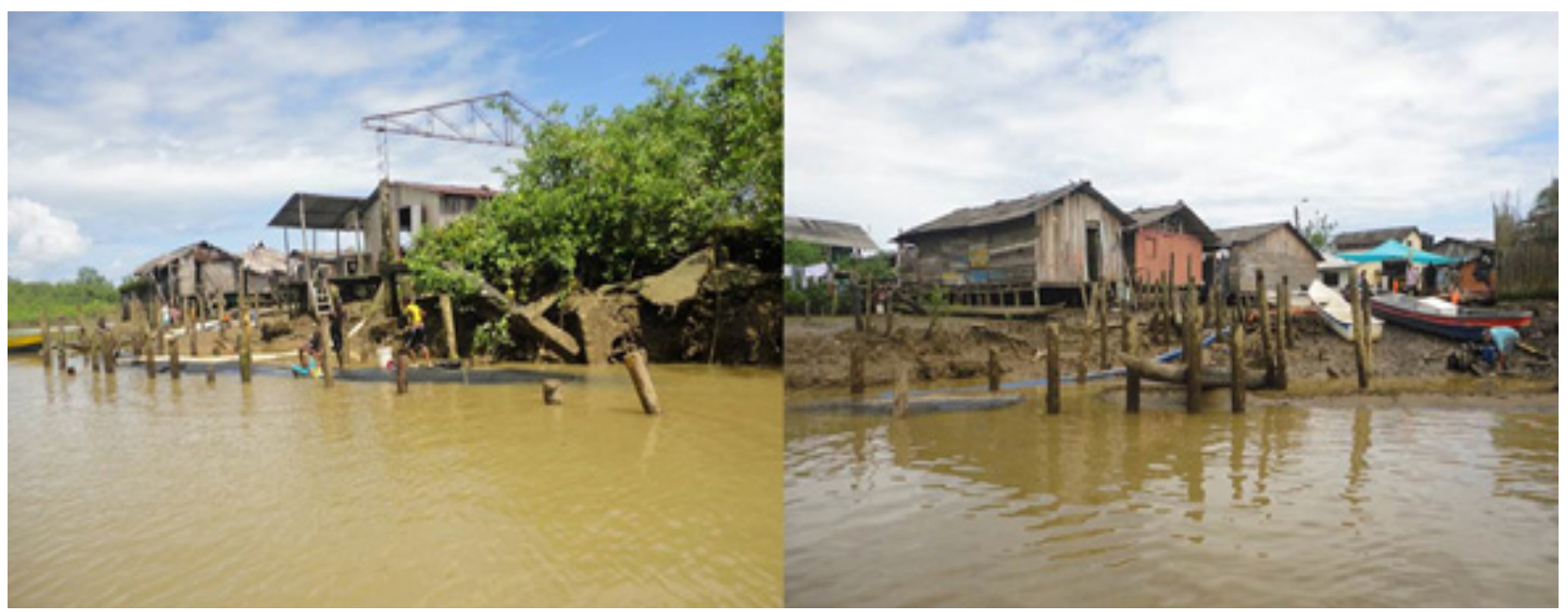

Figura 4. Erosión hídrica sobre el cauce del río Ancachí.

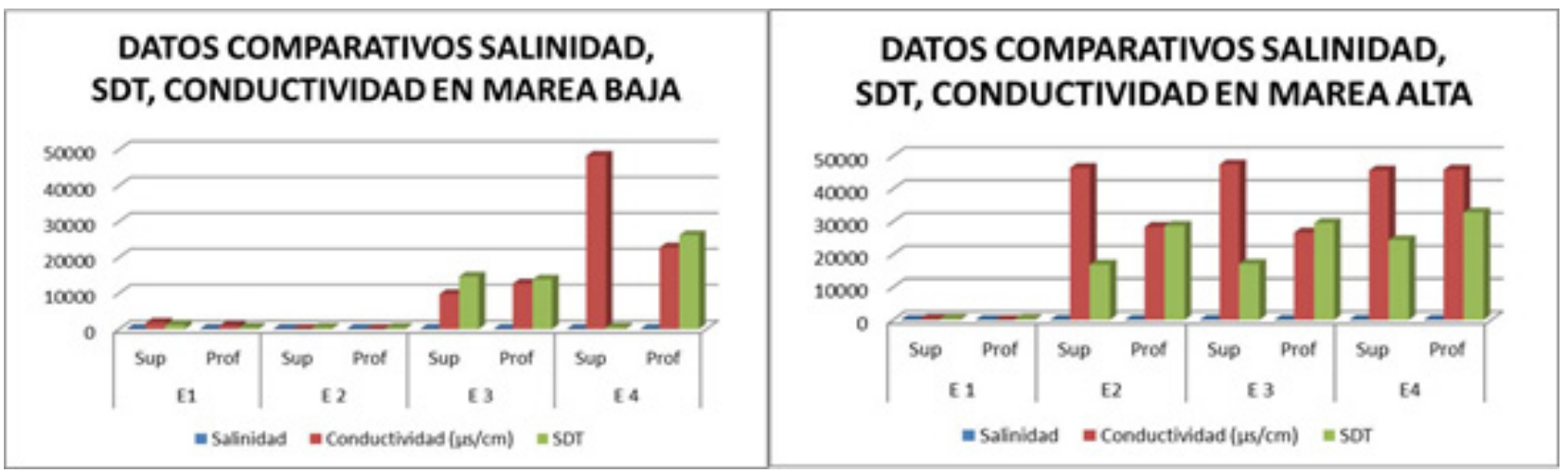

Figura 5. Datos comparativos SDT, conductividad y salinidad en la bahía de Nuquí.

necesaria para la oxidación de los compuestos orgánicos degradables existentes en el líquido residual, es una característica cuantificable del grado de contaminación del agua a partir de su contenido de sustancias biodegradables; este permaneció constante en todos los puntos de muestreo tanto en marea baja como en marea alta, obtenido por debajo de 2 mg/l encontrándose dentro del límite establecido por la normatividad ambiental colombiana (Decreto 1594 de 1984).

En relación con los coliformes fecales, se encontraron entre 17 y 540 NMP, obteniéndose el valor más alto en la profundidad del río Ancachí, lo cual pudo estar determinado por los vertimientos directos del sistema de alcantarillado urbano del municipio realizado a través de un sistema sumergido sobre esta fuente hídrica. Adicionalmente, la estación 2 ubicada en la intercepción de los ríos Ancachí, Nuquí y la zona costera, arrojaron un valor de 220 NMP en la superficie, toda vez que el sistema de alcantarillado presenta múltiples puntos de vertimientos donde cada uno de ellos hace su aporte a la contaminación con aguas residuales provenientes de grupos reducidos de viviendas. A lo anterior, se puede evidenciar que pese a la capacidad de dilución de la bahía, se presenta un aporte significativo de coliformes fecales en la estación 3 , el cual fue reducido a 77 NMP. Se concluye entonces, que de acuerdo con lo dispuesto en la normatividad colombiana, la estación de monitoreo 1 y 2 exceden los niveles permisibles establecidos para la calidad ambiental y de descargas de afluentes (Decreto 1594 de 1984), la cual establece un valor para aguas marinas de 200 NMP/100 ml. Caso similar al de la bahía de Nuquí, se presentó en la bahía de Banderas, Jalisco-Nayarit (México), en la cual de acuerdo con Cortés (2003), las estaciones del río Ameca (E-3) y el río Cuale (E-2) presentan una mayor concentración rebasando en algunos casos, los límites permisibles de acuerdo con la Norma Oficial Mexicana de $>200 \mathrm{NMP} / 100 \mathrm{ml}$. En general, se notó una considerable variación de las bacterias indicadoras desde 3 hasta 24.000 org/100 ml. Esto tiene gran importancia para la determinación de la calidad sanitaria del agua costera de Bahía de Banderas, porque los valores reportados son evidencia de contaminación de origen fecal en el sistema.

En términos generales, las fluctuaciones en las concentraciones de estas bahías, se atribuye a las aguas residuales 


\section{Marea y factores antropogénicos. Z Quesada et al.}

domésticas, las cuales hacen sus descargas al medio marino (Cortés 2003). Lo anterior no difiere de la afirmación hecha por Ramos et al. (2008), quienes manifiestan que en Colombia el problema de la contaminación microbiológica se presenta de manera generalizada, a causa de los vertimientos de aguas residuales en los ambientes marinos y costeros, así como también en otros ecosistemas acuáticos, problemática que se ve magnificada si se tiene en cuenta la falta de tratamiento de los desechos líquidos y sólidos a lo largo de la geografía nacional.

Por otro lado, las concentraciones de grasas y aceites presentaron valores por encima de lo establecido en el mismo, lo cual se atribuye al desarrollo de actividades antrópicas que involucran el manejo de combustibles, como la actividad portuaria, el transporte marítimo, a lo que se suma el aporte de aguas residuales domésticas con altas concentraciones de detergentes y aceites.

En relación con los nutrientes, las fluctuaciones oscilaron entre $30,8 \mathrm{mg} / \mathrm{l}$ y superior a $154.9 \mathrm{mg} / \mathrm{l}$ para el caso de nitratos y entre 0,116 y más de $1,23 \mathrm{mg} / 1$ de nitritos; las variaciones del fosfato osciló entre $0,73 \mathrm{mg} / 1$ y más de $2,75 \mathrm{mg} / \mathrm{l}$ en marea baja. Las mayores concentraciones se obtuvieron en los puntos de monitoreo 2 y 3 ubicados 200 metros después de la intercepción del río Ancachí con la bahía. Los datos registrados muestran un aporte significativo de nutrientes proveniente de las actividades de la zona urbana, los cuales son influidos por las condiciones mareales y las profundidades de las mismas, que para el caso de los nitratos, las concentraciones difieren notablemente tanto para marea alta como para marea baja, lo cual se puede deber a las fluctuaciones de la salinidad, al aporte por los cuerpos de agua dulce (Del Blanco et al. 2014) y a los vertimientos provenientes del sistema de alcantarillado. Sin embargo, de acuerdo con los criterios de calidad de agua internacionales para preservación de la biota acuática específicamente para Panamá, Cooke et al. (2001), señalan que para las variables nitratos y nitritos, los valores permisibles deben estar por debajo de $200 \mathrm{mg} / \mathrm{l}$ y $<0,6 \mathrm{mg} / 1$ respectivamente, evidenciándose así, que las concentraciones obtenidas cumplen con los límites establecidos. Pese a lo anterior, en relación con el fosfato, este se encontró por encima del límite establecido por el Ministerio de Vivienda, Ordenamiento Territorial y Medio Ambiente de Uruguay (2007), el cual no debe superar los 0,025mg/1 para la preservación de la biota acuática.

En términos generales, a pesar de que algunos parámetros como oxígeno disuelto, $\mathrm{pH}$ y la temperatura se encuentran dentro de los rangos establecidos por el Decreto 1594 de 1984 para la conservación de la flora y fauna. La misma se ve restringida por el aporte de grandes concentraciones de parámetros como coliformes fecales, DBO5 y nutrientes como el fosfato, provenientes de las descargas de aguas residuales domésticas, lo que puede representar problemas asociados con la salud pública teniendo en cuenta que el ecosistema costero alberga numerosos bienes y servicios ambientales y ecosistémicos para la zona.

Pese a lo anterior, no se puede atribuir a las descargas de aguas residuales todas las perturbaciones existentes sobre la bahía, porque existen otras posibles causas para la misma como el aporte de sólidos disueltos y sedimentos como consecuencia de la erosión de la ronda hídrica del río Ancachí. Situación similar reportó Guzmán y Norat(2014), en su estudio sobre la calidad microbiológica de las aguas superficiales en las bahías de Mayagüez y Añasco, Puerto Rico, donde se establecieron tres estaciones de monitoreo localizadas en áreas cercanas a las desembocaduras de los ríos Guanajibo (E1), Yagüez (E2) y Añasco (E3), y la estación de monitoreo localizada en el área de descarga de las aguas usadas de la Planta Regional de Tratamiento de Aguas Usadas de Mayagüez a través de un emisario submarino (E4). Las concentraciones mayores de sólidos suspendidos fueron determinadas en las estaciones E1, E2 y E3, las cuales están influidas por el arrastre de sedimentos de los tres ríos que descargan en estas áreas. Por los resultados obtenidos se puede asumir que de estos tres ríos, el río Añasco es el que más contribuye con sedimentos suspendidos en la bahía.

Por otro lado, la influencia de las mareas permiten tener una visión general de la dirección de arrastre de contaminantes, materia orgánica, sólidos suspendidos, entre otros, así como de las aguas provenientes del océano, a su vez de las entradas y salidas de estas con los cambios mareales a lo largo del día (Pinzón et al. 2014). En este sentido los resultados obtenidos en marea baja y marea alta, dan cuenta de la capacidad de dilución de parámetros como los nutrientes los cuales disminuyeron con la entrada de agua de mar a la bahía, además del aporte de sólidos disueltos que entran a la misma por acción de la salinidad proveniente de las mareas altas, lo que se ve reflejado en los valores obtenidos de conductividad. Otros parámetros como el oxígeno disuelto, el pH y la temperatura, se mantienen casi constantes con fluctuaciones mínimas independientemente de las condiciones mareales.

\section{Conclusiones}

El ecosistema costero se evidencia que se encuentra influido por las descargas de vertidos líquidos provenientes de los río Ancachí y Nuquí, lo cual reduce la buena calidad y por lo tanto la productividad del recurso; en este sentido, no solo se ven afectados los organismos al interior de esta sino también la población del municipio, sin dejar de lado que la contaminación de la fuente, es producto de las actividades realizadas por la comunidad de manera 


\section{Bioetnia Volumen 11, 2014}

inadecuada y que ejercen un significativo impacto sobre el sistema hídrico y sobre todo los ríos en mención, siendo estos vulnerables a la contaminación, que provoca una modificación de la composición fisicoquímica del agua y compromete su utilización, al dificultar el proceso natural de autodepuración de los mismos.

En términos generales, en cuanto a las concentraciones de las variables fisicoquímicas, las cuales están influidas por el desarrollo de actividades domésticas en el casco urbano del municipio de Nuquí los parámetros monitoreados no restringen la preservación de la vida acuática y son aptos para la preservación de la biota marina; sin embargo, existen áreas puntuales que de acuerdo con las presiones y perturbaciones urbanas restringen el crecimiento de la misma por el aporte de altas cargas contaminantes relacionadas concoliformes fecales, DQO, DBO5, grasas y aceites en fuentes receptoras de aguas residuales domésticas. Pese a lo anterior, es probable que la dinámica de las corrientes marinas, la capacidad de depuración del mar y la capacidad de dilución de las fuentes hídricas monitoreadas como el río Ancachí y su intercepción con la zona costera, tengan influencia en el comportamiento favorable de las concentraciones de las variables fisicoquímicas registradas; sin embargo, se puede suponer que variables internas como el tiempo, el crecimiento poblacional y el consecuente incremento del desarrollo de actividades socioeconómicas, pueden provocar un aumento en la contaminación por vertimientos líquidos y sólidos en el ecosistema.

\section{Literatura citada}

Aguas y Aguas de Pereira. 2012. Plan de saneamiento y manejo de vertimientos (PSMV) para el municipio de Nuquí. Proyecto Plan de Inversiones todos por el Pacífico, Chocó. Pereira: Aguas y Aguas de Pereira.

Banco de Occidente. 2014. El Chocó Biogeográfico de Colombia. En: Libros de la Colección Ecológica del Banco de Occidente. [fecha de consulta 21 de agosto de 2014]. Disponible en: http://www.imeditores.com/

Boyd C. 1982. Water quality management for pond fish culture. New York: Elsevier; 318 pp.

Burke L, Kura Y, Kassem K, Revenga C, Spalding M, McAllister D. 2001.
Coastal ecosystems. Pilot analysis of global ecosystems. Washington DC: World Resources Institute; $50 \mathrm{pp}$.

Cortés MdelC. 2003. Importancia de los coliformes fecales como indicadores de contaminación en la Franja Litoral de Bahía de Banderas, Jalisco-Nayarit. Centro Universitario de la Costa, Campus Puerto Vallarta, Universidad de Guadalajara, Jalisco, México. Rev Biomed. 14: 121-3.

Cooke R, Griggs J, Sánchez L, Díaz C, Carvajal YD. 2001. Recopilación y presentación de datos de recursos ambientales y culturales en la región occidental de la cuenca del Canal de Panamá. Presentado a la ACP el 8 de noviembre de 2001. Panamá: Consorcio TLBG UP STRI.

Del Blanco L, Asteasuain R, Arlenghi J, Avena J, Marcovecchio J. 2014. Efecto de la marea en la distribución de nutrientes en planicies de marea del estuario de Bahía Blanca. [fecha de consulta: 24 de septiembre de 2014]. Disponible en: http://www.ecopuerto.com/bicentenario/informes/ EFECTOMAREAENCALIDAD.pdf

Derisio JC. 1992. Introdução ao controle da poluição ambiental. São Paulo: CETESB; $201 \mathrm{pp}$.

Garay J, Vélez AM. 2004. Programa Nacional de Investigación, Evaluación, Prevención, Reducción y Control de Fuentes Terrestres y Marinas de Contaminación al Mar (PNICM). Santa Marta: Instituto de Investigaciones Marinas y Costeras “José Benito Vives DeAndréis"(INVEMAR); 110 pp.

GESAMP. 2001. A sea of troubles. IMO/FAO/UNESCO-IOC/ WMO/WHO/ IAEA/UN/UNEP Joint Group of Experts on the scientific Aspects of Marine Environmental Protection (GESAMP). Reports and Studies. 70: 35.

Guzmán-Colón B, Norat-RamírezJ. 2014. Calidad microbiológica de las aguas superficiales en las bahías de Mayagüez y Añasco, Puerto Rico. Departamento de Salud Ambiental, Recinto de Ciencias Médicas, Universidad de Puerto Rico. [fecha de consulta 23 de octubre de 2014]. Disponible en: http://www.bvsde.paho.org/bvsaidis/puertorico/lx.pdf

Instituto de Investigaciones Ambientales del Pacífico(IIAP). 2014. Evaluación fisicoquímica y ecológica las de aguas costeras en la bahía de Nuqui como instrumento de análisis de los aportes contaminantes de las fuentes hídricas continentales urbanas. Informe técnico. Quibdó: IIAP.

Ministerio de Vivienda, Ordenamiento Territorial y Medio Ambiente de Uruguay. 2007. Estándares de calidad de agua para la preservación de la biota acuática. Montevideo: MVOTMAU.

Obeso-Nieblas M, Gaviño-Rodríguez JH, Obeso-Huerta H. 2012. Variabilidad espacial y estacional de temperatura, salinidad y densidad en Bahía Concepción, Golfo de California, México. Rev Biol Mar Oceanograf. 47 (3): 489-502.

Pinzón-Bedoya ML, García-Castellanos AK, Casanova-Rosero RF. 2014. Seguimiento a la influencia de la marea, condiciones meteorológicas y factores antropogénicos sobre la variabilidad diaria de parámetros fisicoquímicos en la bahía de Tumaco (Colombia). [fecha de consulta 23 de octubre de 2014]. Vistua. 5 (2). Disponible en: http://www.unipamplona.edu.co

Ramos-Ortega LM, Vidal LA, Vilardy QS, Saavedra Díaz L. 2008. Análisis de la contaminación microbiológica (coliformes totales y fecales) en la bahía de Santa Marta, Caribe colombiano. Acta Biol Colomb. 13 (3): 87-98.

República de Colombia. Decreto 1594 de 26 de junio de1984. Bogotá: Ministerio de Agricultura. 